\title{
Response to the letter regarding the article "Impact of plant-based diet on lipid risk factors for atherosclerosis"
}

The aim of the study entitled "Impact of plant-based diet on lipid risk factors for atherosclerosis" [1] was to investigate the serum lipid profile, with particular regard to the parameters characterizing the high-density lipoprotein in vegan subjects without subclinical atherosclerosis.

Beyond an assessment of the concentration of individual high-density lipoprotein fractions, we also studied the activity of paraoxonase- 1 and 8 -iso-prostaglandin F2 $\alpha$ concentration to demonstrate the effect of a vegan diet on lipid-connected parameters characterizing the oxidative balance.

We agree that a vegan diet, as an extreme form of diet, may be exposed to an insufficient supply of vitamin B12. Furthermore, vitamin B12 deficiency, through its participation in the metabolism of homocysteine, can be an important factor co-responsible for the development of oxidative stress in the body. At the same time, we agree that an evaluation of serum vitamin B12 and homocysteine level would be beneficial for a better characterization of the study group.

However, we would like to state that the analysis of the food intake questionnaire showed that all vegan subjects used vitamin supplementation, and daily intake of B12 by our vegans was higher than in omnivores. Additionally, we demonstrated no difference in the activity of paraoxonase- 1 and 8-iso-prostaglandin F2 $\alpha$ concentration between vegan and omnivore subjects, which indicates no increased risk of lipids oxidative modification.

In summary, bearing in mind the main aim of the project, the results of the food intake questionnaire and the results of paraoxonase- 1 activity and 8 -iso-prostaglandin F2 $\alpha$ concentration, we decided that the designation of vitamin B12 in the study population may be inconclusive [2].

Conflict of interest: None declared

\section{References}

1. Kuchta A, Lebiedzińska A, Fijałkowski M et al. Impact of plant-based diet on lipid risk factors for atherosclerosis. Cardiol J, 2016; 23: 141-148. doi: 10.5603/CJ.a2016.0002.

2. Cerit L. Plant-based diet and vitamin B12. Cardiol J, 2016; 23: 586. doi: 10.5603/CJ.2016.0080.

Agnieszka Kuchta ${ }^{1}$, Anna Lebiedzinska ${ }^{2}$, Marcin Fijatkowski ${ }^{3}$, Rafat Gataska ${ }^{3}$, Ewelina Kreft ${ }^{4}$, Magdalena Totoń ${ }^{1}$, Kuba Czaja ${ }^{2}$, Anna Koztowska ${ }^{2}$, Agnieszka Ćwiklińska ${ }^{1}$, Barbara Kortas-Stempak ${ }^{1}$, Adrian Strzelecki ${ }^{5}$, Anna Gliwinska ${ }^{1}$,Kamil Dabkowski ${ }^{1}$, Maciej Jankowski ${ }^{1}$

${ }^{1}$ Department of Clinical Chemistry, Medical University of Gdansk, ul.Dębinki 7, 80-211 Gdańsk, Poland, e-mail: agakuchta@gumed.edu.pl

${ }^{2}$ Chair and Department of Bromatology, Medical University of Gdansk, Poland ${ }^{3} 1^{\text {st }}$ Chair and Clinic of Cardiology, Medical University of Gdansk, Poland ${ }^{4}$ Department of Monitored Therapy and Pharmacogenetics, Medical University of Gdansk, Poland

${ }^{5}$ Chair and Clinic of Internal Medicine, Connective Tissue Diseases and Geriatrics, Medical University of Gdansk, Poland 